\title{
Editorial of the evolving and hybrid systems' modelling special issue
}

\author{
Lazaros Iliadis $^{1}$ - Ilias Maglogiannis ${ }^{2}$
}

Published online: 6 August 2020

(c) Springer-Verlag GmbH Germany, part of Springer Nature 2020

Evolving systems are employing inheritance and they perform gradual changes in order to achieve adaptation and continuous "life-long" learning. This is necessary under unknown or dynamic environments which is the typical case in real world. Such systems are employing Fuzzy Logic (FL), advanced Artificial Neural Networks' (ANN) algorithms and moreover hybrid approaches that have the potential to offer optimized solutions for intelligent information representation. This is the Evolving and Hybrid Systems modelling Special Issue, of the Evolving systems (EVOS) Springer journal. It is comprised of contemporary research written by world experts in this emerging scientific field, who present their recent advances in designing algorithms and applying them successfully in real life cases. Emphasis is given in Anomaly Detection and cybersecurity, Natural Language, Biomedical Systems, Ensemble and Optimization techniques. This issue has been developed to form a reference guide for scientists of various domains who are looking for the optimal data mining, engineering and mathematical modelling approaches. Totally eleven papers were accepted to be published.

The first paper has been authored by Georgios Drakopoulos Department of Informatics of the Ionian University, Corfu, Greece, Andreas Kanavos, University of Patras School of Engineering, Patras, Achaia, Greece, Phivos Mylonas, Department of Informatics of the Ionian University, Corfu, Greece, and Spyros Sioutas, Department of Computer Engineering and Informatics, Patras, Achaia, Greece. It is entitled "Discovering Sentiment Potential in Twitter Conversations with Hilbert-Huang Spectrum". This work primarily focuses

Lazaros Iliadis

liliadis@civil.duth.gr

Ilias Maglogiannis

imaglo@unipi.gr

1 School of Engineering, Department of Civil Engineering, Lab of Mathematics and Informatics (ISCE), University Campus, Democritus University of Thrace, Kimmeria, 67100 Xanthi, Greece

2 Department of Digital Systems, University of Piraeus, Karaoli and Dimitriou 80, 18534 Piraeus, Greece on determining which tweets are causing multiple sentiment polarity alternations to occur, based on a window segmentation approach. Moreover, it introduces an offline framework for discovering affective pivot points in a conversation, based on its Hilbert-Huang spectrum. Given that it is highly desirable to track the sentiment shifts of a Twitter conversation while it unfolds, an Adaptive approach is presented for approximating the window sizes obtained by the offline methodology.

The authors of the second paper are Aikaterini Protogerou, University of Macedonia, Thessaloniki, Greece, Stavros Papadopoulos, Anastasios Drosou, and Dimitrios Tzovaras from the Information Technologies InstituteCERTH, Thessaloniki, Greece, and Ioannis Refanidis from University of Macedonia, Thessaloniki, Greece. Its title is "A Graph Neural Network method for distributed Anomaly Detection in IoT". Graph-based anomaly detection solutions, are accommodating the cohesiveness among the involved entities, modeling their interrelations and incorporating their structural, content and temporal attributes. This research introduces a multi-agent system, with each agent implementing a Graph Neural Network, in order to exploit the collaborative and cooperative nature of intelligent agents for anomaly detection. The authors are proposing a distributed detection scheme, aiming to efficiently monitor the entire network's infrastructure. The system is applied against cyber-attacks such as the Distributed Denial-of-Service.

The third paper is entitled "On Solving Single Elevator-like Problems Using a Learning Automata-based Paradigm" and it is written by Omar Ghaleb and John Oommen, Carleton University Ottawa Ontario, Canada. This paper concentrates on a host of problems with characteristics similar to the ones related to moving elevators within a building, known as Elevator-like Problems. Apart from proposing benchmark solutions, this paper has introduced two novel Learning Automata solutions for the single-elevator scenario. The simulation results demonstrate the efficiency of the proposed solutions.

The fourth research paper is entitled "Suicidal Tendencies Prediction in Greek Poetry". It has been authored by Stefanos Nikiforos, Alexandros-Dimitrios Zervopoulos, Evangelos Geramanis, Alexandros Toulakis, Asterios Papamichail, 
Dimitrios Triantafylloy, Theofanis Tasoulas and Katia-Lida Kermanidis, from the Ionian University, Corfu, Greece. Natural language processing has been successfully used to predict a writer's tendency of committing suicide, by considering various text types, namely: suicide notes, microblog posts, lyrics and poems. This paper extends an earlier research effort on text mining in Greek Poetry, by employing more sophisticated approaches. It employs Deep Neural Networks and it considers additional morphosyntactic-semantic features, based on writers' emotions and on Big Five personality traits. Totally, poems of thirteen twentieth century Greek poets are considered.

Stavros Ntalampiras, Universita degli Studi di Milano, Italy and Ilyas Potamitis, Technological Educational Institute of Crete Greece, are the authors of the fifth paper. The title of this research is "Automatic Acoustic Identification of Respiratory Diseases". The general idea is that many diseases affecting the human respiratory system are associated with distinctive sounds. This paper proposes a novel methodology for the automatic identification by means of signal processing and pattern recognition. The introduced system considers a suitable feature set, based on wavelet packet analysis, characterizing data coming from diverse classes of respiratory sounds. The patterns revealed by the feature extraction stage, are modelled by using Hidden Markov models (HMMs). Automatic identification is carried out via a directed acyclic graph scheme limiting the problem space, based on decisions made by the available HMMs.

The sixth paper is by Daniel Perez, Serafin Alonso, Antonio Moran, Miguel Prada and Juan Jose Fuertes, from the University of Leon, Spain. Its title is "Evaluation of feature learning for anomaly detection in network traffic". A variety of well-known anomaly detection algorithms are employed, which address intrusion detection as a semi-supervised problem. Patterns that deviate from a baseline pattern corresponding to normal traffic, are labelled as anomalous. Furthermore, this assessment is performed on four publicly available benchmarks.

Spyridon Papastergiou, University of Piraeus Greece, Haralambos Mouratidis, Centre for Secure, Intelligent and Usable Systems University of Brighton UK, and Stockholm University Sweden, and Eleni-Maria Kalogeraki, University of Piraeus Greece, have authored the seventh paper. Its title is "Handling of Advanced Persistent Threats and Complex Incidents in HealthCare, Transportation and Energy ICT infrastructures". It focuses in the domain of Critical Infrastructure Security, by introducing CyberSANE, a novel dynamic and collaborative warning and response system. It supports security officers and operators to recognize, dynamically analyze, and respond to security threats and risks.

The eighth paper is authored by Michail Spiliotis, Democritus University of Thrace, School of Engineering, Xanthi, Greece. The title of the paper is "A Multicriteria fuzzy pattern recognition approach for assessing the vulnerability to drought: Mediterranean region". The first objective of this research is to develop a novel integrated approach for drought vulnerability estimation, by considering the characteristics of a system that make it prone to be affected by an external hazard. The second objective is to investigate the link between fuzzy pattern recognition and distance based multi-criteria classification. Relevant information is grouped based on drought sensitivity and adaptive capacity criteria. The link with the Multicriteria Technique for Order of Preference by Similarity to Ideal Solution has been investigated.

O. Ekaba Bisong and B. John Oommen, Carleton University Ottawa, Ontario, Canada are the authors of the ninth research paper entitled "On Utilizing an Enhanced Object Partitioning Scheme to Optimize Self-Organizing Lists-on-Lists". This paper is referring to the minimization of retrieval cost, using a Singly-Linked List (SLL) in which the elements are accessed by a Non-stationary Environment, exhibiting the so-called "Locality of Reference". A novel solution to the problem has been introduced, utilizing an Adaptive Data Structure, using composite hierarchical sub-structures. The authors have designed hierarchical Lists-on-Lists by assembling a SLL into a hierarchical scheme that results in "SLLs on SLLs".

The tenth research paper is entitled "On ensemble techniques of weight-constrained neural networks" and it has been authored by ioannis Livieris, University of Patras, Greece, Lazaros Iliadis, Democritus University of Thrace, School of Engineering, Xanthi, Greece and Panagiotis Pintelas, University of Patras, Greece. The authors are introducing two ensemble prediction models based on Bagging and Bossting, which exploit the classification performance of Weight-Constrained Neural Networks. Several benchmarks from the UCI repository have been considered, in order to evaluate the performance of the two proposed models against other state-of-the-art ensemble classifiers.

Lam Gia Thuan, Vietnamese-German University, Vietnam, Doina Logofatu, Frankfurt University of Applied Sciences, Germany and Costin Badica, University of Craiova, Romania are the authors of the eleventh accepted paper. The paper's title is: "A Novel Real-time Design for Fighting Game AI". Real-time fighting games are challenging for computer agents, requiring very powerful machines or state-of-the-art algorithms capable of taking actions in milliseconds. The authors introduce several generic heuristics to be used in combination with Monte-Carlo Tree Search and they compare with Evolutionary Algorithms or Deep Reinforcement Learning approaches.

This EVOS issue comprises of state of the art research papers. We strongly believe that the readers will find insights that will motivate further research in the underline domains.

The Guest Editors. 